\title{
Advances into the Epigenetic Regulation by DNA Methylation in Non-Alcoholic Fatty Liver Disease
}

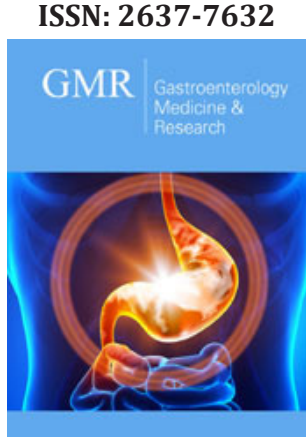

*Corresponding author: Teresa RubioTomás, Institute for Biomedical Research August Pi and Sunyer (IDIBAPS), Spain

Submission: 眥 July 31, 2021

Published: 盢August 12, 2021

Volume 6 - Issue 1

How to cite this article: Teresa RubioTomás. Advances into the Epigenetic Regulation by DNA Methylation in NonAlcoholic Fatty Liver Disease. Gastro Med Res. 6(1). GMR. 000628. 2021. DOI: 10.31031/GMR.2021.06.000628

Copyright@ Teresa Rubio-Tomás, This article is distributed under the terms of the Creative Commons Attribution 4.0 International License, which permits unrestricted use and redistribution provided that the original author and source are credited.

\author{
Teresa Rubio-Tomás ${ }^{1,2 *}$ \\ ${ }^{1}$ Institute for Biomedical Research August Pi and Sunyer (IDIBAPS), Spain \\ ${ }^{2}$ School of Medicine, University of Crete, Greece
}

\begin{abstract}
The incidence of Non-Alcoholic Fatty Liver (NAFLD) and its complications is increasing worldwide, due to different factors, but especially to the increase of hypercaloric diets, sedentary lifestyle and obesity, as well as an increase in the percentage of aged populations in Western countries. Many efforts are being done to understand the pathogenesis of NAFLD to stop this global pandemic and many alterations in epigenetic mechanisms have been detected during the course of the disease. In this mini-review I will focus on recent articles (from the last year) that explore the link between deregulation of DNA methylation, an important epigenetic mechanism, and NAFLD development and progression towards steatohepatitis, cirrhosis and liver cancer.
\end{abstract}

\section{Introduction}

Non-Alcoholic Fatty Liver (NAFLD) is an etiology of chronic liver disease and can progress to steatohepatitis, cirrhosis and liver cancer, is increasing worldwide due to unhealthy lifestyle (i.e., Western diet, lack of physical exercise and subsequent obesity), among other factors. Public health interventions, such as nutritional education and programs dedicated to promote physical activity among all range of age, need to be implemented to prevent or revert the liver damage [1]. Nevertheless, it is also important to unravel the molecular mechanisms governing the development of NAFLD and its progressing to more advanced stages, including cancer [2]. In this sense, the relevance of epigenetics has been recently explored since the importance of epigenetic regulation in healthy liver and liver diseases is nowadays undoubtable $[3,4]$.

\section{DNA methylation in NAFLD}

DNA methylation in NAFLD has been extensively reviewed recently [5]. Here I will describe briefly the most recent data regarding this topic. Hajiri T et al. [6] studied the role of peroxisome proliferator-activated receptor $\gamma$ (PPAR $\gamma$ ) in the liver of mice subjected to high-fat diet, concluding that high-fat diet-induction of ppary DNA promoter demethylation activated the expression of PPAR $\gamma$ and its target genes, vldlr and cd36, promoting excessive lipid accumulation in the liver (hepatic steatosis), therefore contributing to the development of NAFLD [6]. In an attempt to find non-invasive biomarkers for diagnosis of NAFLD, Pan X, et al. [7] performed genome-wide DNA methylation profiling in peripheral blood leukocytes from patients with NAFLD and controls to identify differentially methylated CpG sites. Their results suggest that hypomethylation of PRKCE and SEC14L3 promoters could potentially be used as non-invasive biomarkers for NAFLD Pan X, et al. [7]. Tian et al. also identified alterations in DNA methylome of the liver of patients with liver hepatic steatosis and liver cancer compared to each other and also compared to healthy controls [8]. This type of studies, such as the one from Yoshida et al., help to decipher the DNA methylation aberrations that give rise to NAFLD, but also the ones that activate the progression from NAFLD to liver cancer [9]. 
On the contrary, Moustakas et al. did not observe differences in global DNA methylation in diet induced NAFLD murine models compared to controls [10]. Kim CY et al. [11] observed that, when methyltransferase-3a (DNMT3A) DNMT3A is recruited to lipogenic genes, it represses its expression by DNA methylation. In obese mice and patients with NAFLD, DNMT3A occupancy and DNA methylation at lipogenic genes are low, which leads to high expression of lipogenic genes and hepatic steatosis [11]. Although no methylase or demethylase activity has been described for this molecule, Odd-skipped related 1 (Osr1) seems to be involved in hepatic steatosis by deregulating global DNA methylation, since, after feeding with high-fat diet or chemical induction of liver cancer, Osr1+/- and WT mice showed differences in the DNA methylome of liver cells [12].

\section{Conclusion and Further Directions}

Although there is a link between aberrant DNA methylation, globally and in specific promoters or genes, and presence of hepatic steatosis and NAFLD, further studies are needed to confirm which of the DNA methylation alterations are causative and promote the pathogenesis of NAFLD. Some of the epigenetic regulators can be targetable Sodum N et al. [13], such as EZH2 Lim HJ et al. [14], and there are already compounds that are promising therapies for NAFLD, such as thiamethoxam [15]. Furthermore, dietary interventions can also impact on epigenetic modifications and, therefore, nutraceuticals should be consider as a treatment for liver and other metabolic diseases [16].

\section{References}

1. Diehl Anna M, Farpour-Lambert NJ, Zhao L, Tilg H (2019) Why we need to curb the emerging worldwide epidemic of nonalcoholic fatty liver disease. Nature Metabolism 1(11): 1027-1029.

2. Juanola O, Martínez-López S, Francés R, Gómez-Hurtado I (2021) Non-alcoholic fatty liver disease: Metabolic, genetic, epigenetic and environmental risk factors. International Journal of Environmental Research and Public Health 18(10): 5227.

3. Choudhary NS, Duseja A (2021) Genetic and epigenetic disease modifiers: Non-Alcoholic Fatty Liver Disease (NAFLD) and Alcoholic Liver Disease (ALD). Transl Gastroenterol Hepatol 6: 2.

4. Zhao Z, Meng J, Su R, Zhang J, Chen J, et al. (2020) Epitranscriptomics in liver disease: Basic concepts and therapeutic potential. Journal of Hepatology 73(3): 664-679.
5. Hyun J, Jung Y (2020) DNA methylation in nonalcoholic fatty liver disease. International Journal of Molecular Sciences 21 (21): 8138.

6. Hajri T, Zaiou M, Fungwe TV, Ouguerram K, Besong S (2021) Epigenetic regulation of peroxisome proliferator-activated receptor gamma mediates high-fat diet-induced non-alcoholic fatty liver disease. Cells 10(6): 1355.

7. Pan X, Wu Y, Peng H, Cai X, Hu Z, et al. (2021) Genome-wide DNA methylation profiling in nonalcoholic fatty liver reveals predictive aberrant methylation in PRKCE and $\mathrm{SEC}_{14} \mathrm{~L}_{3}$ promoters. Dig Liver Dis, pp. 00263-00272.

8. Tian Y, Arai E, Makiuchi S, Tsuda N, Kuramoto J, et al. (2020) Aberrant DNA methylation results in altered gene expression in non-alcoholic steatohepatitis-related hepatocellular carcinomas. J Cancer Res Clin Oncol 146(10): 2461-2477.

9. Yoshida T, Tsuchiya A, Kumagai M, Takeuchi S, Nojiri S, et al. (2020) Blocking sphingosine 1-phosphate receptor 2 accelerates hepatocellular carcinoma progression in a mouse model of NASH. Biochemical and Biophysical Research Communications 530(4): 665-672.

10. Moustakas Ioannis I, Katsarou A, Ioanna Legaki A, Pyrina I, Ntostoglou $\mathrm{K}$, et al. (2021) Hepatic senescence accompanies the development of NAFLD in non-aged mice independently of obesity. International Journal of Molecular Sciences 22(7): 3446.

11. Kim CY, Seok S, Zhang Y, Ma J, Kong B, et al. (2020) Intestinal FGF15/19 physiologically repress hepatic lipogenesis in the late fed-state by activating SHP and DNMT ${ }_{3}$ A. Nature Communications 11 (1): 5969.

12. Zhou Y, Liu Z, Lynch EC, He L, Cheng H, et al. (2021) Osr1 regulates hepatic inflammation and cell survival in the progression of non-alcoholic fatty liver disease. Laboratory Investigation 101(4): 477-489.

13. Sodum N, Kumar G, Bojja SL, Kumar N, Mallikarjuna Rao C (2021) Epigenetics in NAFLD/NASH: Targets and therapy. Pharmacol Res 167: 105484.

14. Lim HJ, Kim M (2020) EZH2 as a potential target for NAFLD therapy. Int J Mol Sci 21(22): 1-15.

15. Yang D, Zhang X, Yue L, Hu X, Wei X, et al. (2021) Thiamethoxam induces nonalcoholic fatty liver disease in mice via methionine metabolism disturb via nicotinamide N-methyltransferase overexpression 273: 129727.

16. Asif S, Morrow NM, Mulvihill EE, Kim KH (2020) Understanding dietary intervention-mediated epigenetic modifications in metabolic diseases. Frontiers in Genetics 11: 590369. 